OCU-PHYS 207

hep-th/0402199

\title{
New Infinite Series of Einstein Metrics on Sphere Bundles from AdS Black Holes
}

\author{
Yoshitake Hashimoto ${ }^{\dagger}$, Makoto Sakaguchi` and Yukinori Yasui* \\ $\dagger$ Department of Mathematics, Osaka City University \\ * Osaka City University Advanced Mathematical Institute (OCAMI) \\ * Department of Physics, Osaka City University \\ Sumiyoshi, Osaka 558-8585, JAPAN
}

\begin{abstract}
A new infinite series of Einstein metrics is constructed explicitly on $S^{2} \times S^{3}$, and the non-trivial $S^{3}$-bundle over $S^{2}$, containing infinite numbers of inhomogeneous ones. They appear as a certain limit of a nearly extreme 5-dimensional AdS Kerr black hole. In the special case, the metrics reduce to the homogeneous Einstein metrics studied by Wang and Ziller. We also construct an inhomogeneous Einstein metric on the non-trivial $S^{d-2}$-bundle over $S^{2}$ from a $d$-dimensional AdS Kerr black hole. Our construction is a higher dimensional version of the method of Page, which gave an inhomogeneous Einstein metric on $\mathbb{C} P^{2} \sharp \overline{\mathbb{C} P^{2}}$.
\end{abstract}

\footnotetext{
†hashimot@sci.osaka-cu.ac.jp

*msakaguc@sci.osaka-cu.ac.jp

*yasui@sci.osaka-cu.ac.jp
} 


\section{Introduction}

Anti-de Sitter (AdS) spaces have attracted renewed interests after the AdS/CFT correspondence conjecture [1, which relates the properties of the supergravity on AdS and those of the strongly coupled gauge theory on the AdS boundary. For example, the Hawking-Page phase transition [2] between AdS and AdS Schwarzschild black holes was interpreted [3] as the phase transition between confining and deconfining phases of the dual gauge theory. Motivated by this, the study of AdS black holes has been extended in various directions. Among them, AdS Kerr black holes with two angular momenta in 5dimensions, as well as ones with one angular momentum in $d$-dimensions were constructed in 4 .

On the other hand, an inhomogeneous Einstein metric on $\mathbb{C} P^{2} \sharp \overline{\mathbb{C} P^{2}}$ was constructed by Page [5]. This metric is of cohomogeneity one with principal orbits $S^{3}$. It was obtained as a certain limit of the 4-dimensional de Sitter black hole together with the Wick rotation. It should be emphasized that this metric is the first example of inhomogeneous Einstein metrics. Furthermore, Böhm proved the existence of an infinite series of Einstein metrics of cohomogeneity one with positive scalar curvature on $S^{N}(5 \leq N \leq 9)$ and $S^{N_{1}+1} \times S^{N_{2}}$ $\left(5 \leq N_{1}+N_{2}+1 \leq 9, N_{1}>1, N_{2}>1\right)[6]$.

Combining these two observations, we explicitly construct new Einstein metrics with positive scalar curvature on sphere bundles, applying the method developed in [5] to the 5-dimensional AdS Kerr black hole with two angular momenta and the $d$-dimensional AdS Kerr black hole with one angular momentum constructed in [4. In summary, we will construct

- an infinite series of Einstein metrics on $S^{2} \times S^{3}$ and $S^{2} \widetilde{\times} S^{3}$ (the non-trivial $S^{3}$-bundle over $\left.S^{2}\right)$ parameterized by a pair of integers $\left(k_{1}, k_{2}\right)$. The bundle type depends on the parity of $k_{1}+k_{2}$; It is trivial if $k_{1}+k_{2}$ is even, and is non-trivial otherwise. When $k_{1} \neq k_{2}$, the metrics are inhomogeneous (see Theorem 1). When $k_{1}=k_{2}$, they are homogeneous Einstein metrics on $S^{2} \times S^{3}$ (see Theorem 2).

- an inhomogeneous Einstein metric on $S^{2} \widetilde{\times} S^{d-2}$, the non-trivial $S^{d-2}$-bundle over $S^{2}$ (see Theorem 3).

It should be noticed that the metrics on $S^{2} \widetilde{\times} S^{3}$ in Theorem 1 obviously are not included in the case of Böhm's existence theorem and that the metrics on $S^{2} \times S^{3}$ are apparently different from the ones which are proved to exist in [6]. The metrics in Theorem 2 coincide with the homogeneous Einstein metrics on $M_{k, 1}^{1,1}$, circle bundles over $\mathbb{C} P^{1} \times \mathbb{C} P^{1}$ studied by Wang and Ziller [7]. The metric in Theorem 3 is of cohomogeneity one with principal orbits $S^{3} \times S^{d-4}$ if $d \geq 5$. In the case of $d=4$, it reproduces the Page metric on $S^{2} \widetilde{\times} S^{2}=\mathbb{C} P^{2} \sharp \overline{\mathbb{C} P^{2}}$ with principal orbits $S^{3}$. 
This paper is organized as follows. In section 2, we apply the method [5] to the 5dimensional AdS Kerr black hole with two angular momenta, and obtain the first class of the Einstein metrics. We will derive the second class of the Einstein metrics from the $d$-dimensional AdS Kerr black hole [4] with one angular momentum in section 3.

\section{5-dimensional Einstein Metrics}

The 5-dimensional Euclidean de Sitter (dS) Kerr metric may be extracted from the Lorentzian AdS Kerr metric [4] by the substitution $t \mapsto-i \tau, \quad a \mapsto-i \alpha, \quad b \mapsto-i \beta$ with $l \mapsto-i l(\mathrm{AdS} \mapsto \mathrm{d} S)$,

$$
\begin{aligned}
g_{5}= & \frac{\Delta_{r}}{\rho^{2}}\left[d \tau-\frac{\alpha \sin ^{2} \theta}{\Xi_{\alpha}} d \phi-\frac{\beta \cos ^{2} \theta}{\Xi_{\beta}} d \psi\right]^{2}+\frac{\Delta_{\theta} \sin ^{2} \theta}{\rho^{2}}\left[\alpha d \tau+\frac{r^{2}-\alpha^{2}}{\Xi_{\alpha}} d \phi\right]^{2} \\
& +\frac{\Delta_{\theta} \cos ^{2} \theta}{\rho^{2}}\left[\beta d \tau+\frac{r^{2}-\beta^{2}}{\Xi_{\beta}} d \psi\right]^{2} \\
& -\frac{1-r^{2} l^{2}}{r^{2} \rho^{2}}\left[\alpha \beta d \tau+\frac{\beta\left(r^{2}-\alpha^{2}\right) \sin ^{2} \theta}{\Xi_{\alpha}} d \phi+\frac{\alpha\left(r^{2}-\beta^{2}\right) \cos ^{2} \theta}{\Xi_{\beta}} d \psi\right]^{2} \\
& +\frac{\rho^{2}}{\Delta_{r}} d r^{2}+\frac{\rho^{2}}{\Delta_{\theta}} d \theta^{2},
\end{aligned}
$$

where

$$
\begin{aligned}
\rho^{2} & =r^{2}-\alpha^{2} \cos ^{2} \theta-\beta^{2} \sin ^{2} \theta, \\
\Delta_{r} & =\frac{1}{r^{2}}\left(r^{2}-\alpha^{2}\right)\left(r^{2}-\beta^{2}\right)\left(1-r^{2} l^{2}\right)-2 M, \\
\Delta_{\theta} & =1-\alpha^{2} l^{2} \cos ^{2} \theta-\beta^{2} l^{2} \sin ^{2} \theta
\end{aligned}
$$

with parameters $\Xi_{\alpha}=1-\alpha^{2} l^{2}$ and $\Xi_{\beta}=1-\beta^{2} l^{2}$. The radii of the horizons are given by the roots of $\Delta_{r}=0$. If there is a double root $r_{0}$, the black hole becomes an extreme black hole, in which the number of the free parameters reduces. The parameters $r_{0}, M, \Xi_{\alpha}$ and $\Xi_{\beta}$ are written in terms of $l$ and the dimensionless parameters

$$
\nu_{1}=\alpha / r_{0}, \quad \nu_{2}=\beta / r_{0},
$$

$\mathrm{as}^{\ddagger}$

$$
\begin{aligned}
r_{0} & =\left[\frac{1-\nu_{1}^{2} \nu_{2}^{2}}{2-\nu_{1}^{2}-\nu_{2}^{2}}\right]^{1 / 2} l^{-1} \\
M_{0} & =\frac{\left(1-\nu_{1}^{2}\right)^{2}\left(1-\nu_{2}^{2}\right)^{2}\left(1-\nu_{1}^{2} \nu_{2}^{2}\right)}{2\left(2-\nu_{1}^{2}-\nu_{2}^{2}\right)^{2}} l^{-2}, \\
\Xi_{\alpha}^{0} & =1-\frac{\nu_{1}^{2}\left(1-\nu_{1}^{2} \nu_{2}^{2}\right)}{2-\nu_{1}^{2}-\nu_{2}^{2}} \\
\Xi_{\beta}^{0} & =1-\frac{\nu_{2}^{2}\left(1-\nu_{1}^{2} \nu_{2}^{2}\right)}{2-\nu_{1}^{2}-\nu_{2}^{2}}
\end{aligned}
$$

\footnotetext{
${ }^{\ddagger}$ The suffix 0 is added for extreme parameters.
} 
In terms of these parameters, we have

$$
\begin{aligned}
\Delta_{r} & =-\left(r-r_{0}\right)^{2} \tilde{\Delta}(r), \\
\tilde{\Delta}(r) & =\frac{1}{r^{2}}\left(r+r_{0}\right)^{2}\left(l^{2} r^{2}-\nu_{1}^{2} \nu_{2}^{2}\right) .
\end{aligned}
$$

Let us consider a nearly extreme black hole; it has two horizons located at $r=r_{1} \equiv$ $r_{0}-\varepsilon$ and $r=r_{2} \equiv r_{0}+\varepsilon$, where the parameter $\varepsilon$ represents a small deviation from the extreme black hole. In the region between two horizons, $\Omega(\varepsilon)=\left\{r \mid r_{1} \leq r \leq r_{2}\right\}$, we introduce a new radial coordinate $\chi(0 \leq \chi \leq \pi)$ by

$$
r=r_{0}-\varepsilon \cos \chi
$$

The $\Delta_{r}=-\left(r-r_{1}\right)\left(r-r_{2}\right) \tilde{\Delta}(r)$ restricted to $\Omega(\varepsilon)$ is then given by

$$
\Delta_{r}=\varepsilon^{2} \tilde{\Delta}\left(r_{0}\right) \sin ^{2} \chi+O\left(\varepsilon^{3}\right)
$$

where

$$
\tilde{\Delta}\left(r_{0}\right)=\frac{4\left(1+\nu_{1}^{2} \nu_{2}^{4}+\nu_{1}^{4} \nu_{2}^{2}-3 \nu_{1}^{2} \nu_{2}^{2}\right)}{2-\nu_{1}^{2}-\nu_{2}^{2}} .
$$

We consider the limit $\varepsilon \rightarrow 0$ of the metric (2.1) in the region $\Omega(\varepsilon)$. In this limit, the term proportional to $\Delta_{r}$ vanishes and so leads to a singularity of the metric. To avoid this, we define a rescaled time coordinate

$$
\eta=\frac{\varepsilon \tilde{\Delta}\left(r_{0}\right)}{r_{0}^{2}\left(1-\nu_{1}^{2}\right)\left(1-\nu_{2}^{2}\right)} \tau+O\left(\varepsilon^{2}\right) .
$$

It is also convenient to define the azimuthal angles

$$
\phi_{1}=\phi+\frac{\alpha \Xi_{\alpha}}{r_{1}^{2}-\alpha^{2}} \tau, \quad \phi_{2}=\psi+\frac{\beta \Xi_{\beta}}{r_{1}^{2}-\beta^{2}} \tau .
$$

The new coordinates $\left(\eta, \chi, \phi_{1}, \phi_{2}, \theta\right)$ are then well-behaved local coordinates in the limit $\varepsilon \rightarrow 0:$

$$
\begin{array}{ll}
\text { (a) } & \frac{\Delta_{r}}{\rho^{2}}\left[d \tau-\frac{\alpha \sin ^{2} \theta}{\Xi_{\alpha}} d \phi-\frac{\beta \cos ^{2} \theta}{\Xi_{\beta}} d \psi\right]^{2} \rightarrow \frac{\rho_{0}^{2}}{\tilde{\Delta}\left(r_{0}\right)} \sin ^{2} \chi d \eta^{2} \\
\text { (b) } & \frac{\Delta_{\theta} \sin ^{2} \theta}{\rho^{2}}\left[\alpha d \tau+\frac{r^{2}-\alpha^{2}}{\Xi_{\alpha}} d \phi\right]^{2} \\
& \rightarrow \frac{r_{0}^{4}\left(1-\nu_{1}^{2}\right)^{2}}{\rho_{0}^{2}} \Delta_{\theta}^{0} \sin ^{2} \theta\left[\frac{d \phi_{1}}{\Xi_{\alpha}^{0}}-\frac{4 \nu_{1}\left(1-\nu_{2}^{2}\right)}{\left(1-\nu_{1}^{2}\right) \tilde{\Delta}\left(r_{0}\right)} \sin ^{2} \frac{\chi}{2} d \eta\right]^{2} \\
\text { (c) } & \frac{\Delta_{\theta} \cos ^{2} \theta}{\rho^{2}}\left[\beta d \tau+\frac{r^{2}-\beta^{2}}{\Xi_{\beta}} d \psi\right]^{2} \\
& \rightarrow \frac{r_{0}^{4}\left(1-\nu_{2}^{2}\right)^{2}}{\rho_{0}^{2}} \Delta_{\theta}^{0} \cos ^{2} \theta\left[\frac{d \phi_{2}}{\Xi_{\beta}^{0}}-\frac{4 \nu_{2}\left(1-\nu_{1}^{2}\right)}{\left(1-\nu_{2}^{2}\right) \tilde{\Delta}\left(r_{0}\right)} \sin ^{2} \frac{\chi}{2} d \eta\right]^{2}
\end{array}
$$




$$
\begin{aligned}
& \text { (d) } \frac{1-r^{2} l^{2}}{r^{2} \rho^{2}}\left[\alpha \beta d \tau+\frac{\beta\left(r^{2}-\alpha^{2}\right) \sin ^{2} \theta}{\Xi_{\alpha}} d \phi+\frac{\alpha\left(r^{2}-\beta^{2}\right) \cos ^{2} \theta}{\Xi_{\beta}} d \psi\right]^{2} \\
& \rightarrow \frac{r_{0}^{4}\left(1-\nu_{1}^{2}\right)\left(1-\nu_{2}^{2}\right)}{\rho_{0}^{2}\left(2-\nu_{1}^{2}-\nu_{2}^{2}\right)}\left[\frac{\nu_{2}\left(1-\nu_{1}^{2}\right)}{\Xi_{\alpha}^{0}} \sin ^{2} \theta d \phi_{1}+\frac{\nu_{1}\left(1-\nu_{2}^{2}\right)}{\Xi_{\beta}^{0}} \cos ^{2} \theta d \phi_{2}\right. \\
& \left.-\frac{4 \nu_{1} \nu_{2} \rho_{0}^{2}}{\tilde{\Delta}\left(r_{0}\right) r_{0}^{2}} \sin ^{2} \frac{\chi}{2} d \eta\right]^{2} \\
& \text { (e) } \quad \frac{\rho^{2}}{\Delta_{r}} d r^{2} \rightarrow \frac{\rho_{0}^{2}}{\tilde{\Delta}\left(r_{0}\right)} d \chi^{2} \\
& \text { (f) } \quad \frac{\rho^{2}}{\Delta_{\theta}} d \theta^{2} \rightarrow \frac{\rho_{0}^{2}}{\Delta_{\theta}^{0}} d \theta^{2}
\end{aligned}
$$

where

$$
\begin{aligned}
\rho_{0}^{2} & =r_{0}^{2}\left(1-\nu_{1}^{2} \cos ^{2} \theta-\nu_{2}^{2} \sin ^{2} \theta\right), \\
\Delta_{\theta}^{0} & =1-\frac{1-\nu_{1}^{2} \nu_{2}^{2}}{2-\nu_{1}^{2}-\nu_{2}^{2}}\left(\nu_{1}^{2} \cos ^{2} \theta+\nu_{2}^{2} \sin ^{2} \theta\right) .
\end{aligned}
$$

Finally, we obtain a metric with two parameters $\nu_{1}$ and $\nu_{2}$

$$
g=h^{2}(\theta) d \theta^{2}+\sum_{i, j=1}^{2} a_{i j}(\theta) \omega^{i} \otimes \omega^{j}+b^{2}(\theta) g_{S^{2}} .
$$

Here, $g_{S^{2}}$ represents the standard metric on $S^{2}$,

$$
g_{S^{2}}=d \chi^{2}+\sin ^{2} \chi d \eta^{2} .
$$

The 1-forms $\omega^{i}(i=1,2)$ are defined by

$$
\omega^{i}=d \psi_{i}+k_{i} \cos \chi d \eta,
$$

where

$$
\begin{aligned}
& k_{1}=\frac{4 \nu_{1}\left(1-\nu_{2}^{2}\right) \Xi_{\alpha}^{0}}{\left(1-\nu_{1}^{2}\right) \tilde{\Delta}\left(r_{0}\right)}=\frac{\nu_{1}\left(1-\nu_{2}^{2}\right)\left(2-\nu_{2}^{2}-\nu_{1}^{2} \nu_{2}^{2}\right)}{1+\nu_{1}^{4} \nu_{2}^{2}+\nu_{1}^{2} \nu_{2}^{4}-3 \nu_{1}^{2} \nu_{2}^{2}}, \\
& k_{2}=\frac{4 \nu_{2}\left(1-\nu_{1}^{2}\right) \Xi_{\beta}^{0}}{\left(1-\nu_{2}^{2}\right) \tilde{\Delta}\left(r_{0}\right)}=\frac{\nu_{2}\left(1-\nu_{1}^{2}\right)\left(2-\nu_{1}^{2}-\nu_{1}^{2} \nu_{2}^{2}\right)}{1+\nu_{1}^{4} \nu_{2}^{2}+\nu_{1}^{2} \nu_{2}^{4}-3 \nu_{1}^{2} \nu_{2}^{2}},
\end{aligned}
$$

and $\psi_{i}$ are introduced as

$$
\phi_{i}=\frac{1}{2}\left(\psi_{i}+k_{i} \eta\right) .
$$

The metric components are found to be $\mathrm{e}^{\S}$

$$
h^{2}=\frac{1-\nu_{1}^{2} \cos ^{2} \theta-\nu_{2}^{2} \sin ^{2} \theta}{\Delta_{\theta}^{0}},
$$

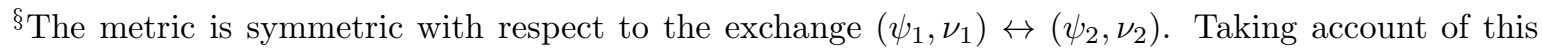
symmetry, we often discuss only the one side. 


$$
\begin{aligned}
a_{11} & =\left(\frac{1-\nu_{1}^{2}}{2 \Xi_{\alpha}^{0}}\right)^{2} \frac{\sin ^{2} \theta}{1-\nu_{1}^{2} \cos ^{2} \theta-\nu_{2}^{2} \sin ^{2} \theta}\left[\Delta_{\theta}^{0}-\frac{\nu_{2}^{2}\left(1-\nu_{1}^{2}\right)\left(1-\nu_{2}^{2}\right)}{2-\nu_{1}^{2}-\nu_{2}^{2}} \sin ^{2} \theta\right] \\
a_{22} & =\left(\frac{1-\nu_{2}^{2}}{2 \Xi_{\beta}^{0}}\right)^{2} \frac{\cos ^{2} \theta}{1-\nu_{1}^{2} \cos ^{2} \theta-\nu_{2}^{2} \sin ^{2} \theta}\left[\Delta_{\theta}^{0}-\frac{\nu_{1}^{2}\left(1-\nu_{1}^{2}\right)\left(1-\nu_{2}^{2}\right)}{2-\nu_{1}^{2}-\nu_{2}^{2}} \cos ^{2} \theta\right], \\
a_{12} & =-\left(\frac{\nu_{1} \nu_{2}\left(1-\nu_{1}^{2}\right)^{2}\left(1-\nu_{2}^{2}\right)^{2}}{4 \Xi_{\alpha}^{0} \Xi_{\beta}^{0}\left(2-\nu_{1}^{2}-\nu_{2}^{2}\right)}\right) \frac{\sin ^{2} \theta \cos ^{2} \theta}{1-\nu_{1}^{2} \cos ^{2} \theta-\nu_{2}^{2} \sin ^{2} \theta}, \\
b^{2} & =\frac{1-\nu_{1}^{2} \cos ^{2} \theta-\nu_{2}^{2} \sin ^{2} \theta}{\tilde{\Delta}\left(r_{0}\right)} .
\end{aligned}
$$

It is straightforward to calculate the Ricci curvature. We find that (2.19) is the Einstein metric with the scalar curvature $20\left(1-\nu_{1}^{2} \nu_{2}^{2}\right) /\left(2-\nu_{1}^{2}-\nu_{2}^{2}\right)$. In the following, we shall consider three cases for two parameters $\nu_{1}$ and $\nu_{2}$;

Case $\mathbf{A} \quad \nu_{1}^{2}, \nu_{2}^{2}>1$ and $\nu_{1}^{2} \neq \nu_{2}^{2}$,

Case B $0 \leq \nu_{1}^{2}, \nu_{2}^{2} \leq 1$ and $\nu_{1}^{2} \neq \nu_{2}^{2}$,

Case C $\quad \nu_{1}^{2}=\nu_{2}^{2} \equiv \nu$.

These conditions ensure that singularities of the Riemannian curvature disappear, and further the metric components are non-negative, i.e., the eigenvalues of $a_{i j}$ are non-negative and $h^{2}, b^{2}>0$.

Next, we consider the condition to avoid orbifold singularities, which restricts the range of the angles $\left(\theta, \psi_{i}\right)$ and the parameters $k_{i}$. We calculate the determinant

$$
\operatorname{det}\left(a_{i j}\right)=\left(\frac{\left(1-\nu_{1}^{2}\right)\left(1-\nu_{2}^{2}\right)}{4 \Xi_{\alpha}^{0} \Xi_{\beta}^{0}}\right)^{2} \frac{\sin ^{2} \theta \cos ^{2} \theta \Delta_{\theta}^{0}}{1-\nu_{1}^{2} \cos ^{2} \theta-\nu_{2}^{2} \sin ^{2} \theta} .
$$

Now clearly $\theta=0, \pi / 2$ are zeros of the determinant and so the range of $\theta$ must be restricted to $0 \leq \theta \leq \pi / 2$. The singularities at $\theta=0, \pi / 2$ are removable bolt singularities. Indeed, near the boundaries the metric behaves as

$$
g \rightarrow \frac{1-\nu_{1}^{2}}{\Xi_{\alpha}^{0}}\left(d \theta^{2}+\frac{1}{4} \theta^{2} d \psi_{1}^{2}\right)+g_{L_{k_{2}}} \text { for } \theta \rightarrow 0,
$$

and

$$
g \rightarrow \frac{1-\nu_{2}^{2}}{\Xi_{\beta}^{0}}\left(d\left(\frac{\pi}{2}-\theta\right)^{2}+\frac{1}{4}\left(\frac{\pi}{2}-\theta\right)^{2} d \psi_{2}^{2}\right)+g_{L_{k_{1}}} \quad \text { for } \quad \theta \rightarrow \frac{\pi}{2}
$$

where

$$
\begin{aligned}
& g_{L_{k_{1}}}=a_{11}(\pi / 2)\left(d \psi_{1}+k_{1} \cos \chi d \eta\right)^{2}+b^{2}(\pi / 2) g_{S^{2}} \\
& g_{L_{k_{2}}}=a_{22}(0)\left(d \psi_{2}+k_{2} \cos \chi d \eta\right)^{2}+b^{2}(0) g_{S^{2}}
\end{aligned}
$$

Thus these singularities are removable provided that the ranges of $\psi_{i}(i=1,2)$ are chosen to be $0 \leq \psi_{i} \leq 4 \pi$. In this range, $\left(\theta, \psi_{1} / 2\right)$ as $\theta \rightarrow 0$ and $\left(\pi / 2-\theta, \psi_{2} / 2\right)$ as $\theta \rightarrow \pi / 2$ are 
the usual polar coordinates on $\mathbb{R}^{2}$. We also demand that $k_{i}$ is integral (see eqn.(2.22) ), then the 1 -forms $\omega_{i}=d \psi_{i}+k_{i} \cos \chi d \eta$ are identified with connections on the lens spaces $L_{k_{i}}=L\left(k_{i}, 1\right)=S^{3} / \mathbb{Z}_{k_{i}}$, and each $k_{i}$ represents the first Chern number (or the monopole charge) as a circle bundle on $S^{2}$. This yields that the manifolds near the boundaries are $\mathbb{R}^{2} \times L_{k_{i}}$, which collapses onto $\{$ point $\} \times L_{k}$ at the boundaries.

Remark 1. There exists a nontrivial root of $\Delta_{\theta}^{0}=0$,

$$
\theta=\theta_{0}, \quad \cos ^{2} \theta_{0}=\frac{2-\nu_{1}^{2}-2 \nu_{2}^{2}+\nu_{1}^{2} \nu_{2}^{4}}{\left(1-\nu_{1}^{2} \nu_{2}^{2}\right)\left(\nu_{1}^{2}-\nu_{2}^{2}\right)}
$$

other than $\theta=0$ and $\pi / 2$. In order to avoid curvature singularities, we restrict $\theta_{0}$ to be in the region $0<\theta_{0}<\pi / 2$ and the parameters $\left(\nu_{1}, \nu_{2}\right)$ in the following region:

Case D $\quad \nu_{2}^{2}>1, \nu_{2}^{2}\left(1+\nu_{1}^{2}\right)<2 \quad$ or $\quad \nu_{2}^{2}<1, \nu_{2}^{2}\left(1+\nu_{1}^{2}\right)>2$,

Case $\mathbf{E} \quad \nu_{1}^{2}>1, \nu_{1}^{2}\left(1+\nu_{2}^{2}\right)<2 \quad$ or $\quad \nu_{1}^{2}<1, \nu_{1}^{2}\left(1+\nu_{2}^{2}\right)>2$.

Then, the metric is regular if we choose the range as $0 \leq \theta \leq \theta_{0}$ for the case $\mathrm{D}$, and $\theta_{0} \leq \theta \leq \pi / 2$ for the case E. However one can not resolve the orbifold singularity at the boundary $\theta=\theta_{0}$ like the cases $\mathrm{A}, \mathrm{B}$ and $\mathrm{C}$.

Having constructed the Einstein metric locally, we now proceed to the global issue for the cases $\mathrm{A}, \mathrm{B}$ and $\mathrm{C}$. It is known that there are two inequivalent classes of $S^{N}$-bundles over $S^{2}$. The $S^{N}$-bundles over $S^{2}=D_{+} \cup D_{-}\left(D_{ \pm}\right.$denote hemispheres $)$are obtained by attaching $D_{+} \times S^{N}$ and $D_{-} \times S^{N}$ as

$$
\begin{aligned}
& (x, \xi) \sim(x, \gamma(x) \xi), \\
& x \in D_{+} \cap D_{-}=S^{1}, \quad \gamma: S^{1} \mapsto S O(N+1) .
\end{aligned}
$$

They are classified by $[\gamma] \in \pi_{1}(S O(N+1))=\mathbb{Z}_{2}$. Globally our metrics (2.19) can be regarded as those on $S^{3}$-bundles over $S^{2}$.

We will discuss the cases $\mathrm{A}, \mathrm{B}$ and $\mathrm{C}$ separately below.

\section{Case A}

We can write the metric (2.19) with $(2.20)-(2.28)$ as

$$
g_{\nu_{1} \nu_{2}}=h^{2}(\theta) d \theta^{2}+\sum_{i, j=1}^{2} a_{i j}(\theta) \omega^{i} \otimes \omega^{j}+b^{2}(\theta) g_{S^{2}}
$$

where

$$
\begin{aligned}
h^{2} & =\frac{1-\nu_{1}^{2} \cos ^{2} \theta-\nu_{2}^{2} \sin ^{2} \theta}{1-\mu_{1}^{2} \cos ^{2} \theta-\mu_{2}^{2} \sin ^{2} \theta} \\
a_{11} & =\frac{1}{4}\left(\frac{\nu_{1}\left(1-\nu_{2}^{2}\right)\left(2-\nu_{1}^{2}-\nu_{2}^{2}\right)}{1+\nu_{1}^{4} \nu_{2}^{2}+\nu_{1}^{2} \nu_{2}^{4}-3 \nu_{1}^{2} \nu_{2}^{2}}\right)^{2} \frac{\left(1-\mu_{1}^{2} \cos ^{2} \theta-\nu_{2}^{2} \sin ^{2} \theta\right) \sin ^{2} \theta}{1-\nu_{1}^{2} \cos ^{2} \theta-\nu_{2}^{2} \sin ^{2} \theta}
\end{aligned}
$$




$$
\begin{aligned}
a_{22} & =\frac{1}{4}\left(\frac{\nu_{2}\left(1-\nu_{1}^{2}\right)\left(2-\nu_{1}^{2}-\nu_{2}^{2}\right)}{1+\nu_{1}^{4} \nu_{2}^{2}+\nu_{1}^{2} \nu_{2}^{4}-3 \nu_{1}^{2} \nu_{2}^{2}}\right)^{2} \frac{\left(1-\nu_{1}^{2} \cos ^{2} \theta-\mu_{2}^{2} \sin ^{2} \theta\right) \cos ^{2} \theta}{1-\nu_{1}^{2} \cos ^{2} \theta-\nu_{2}^{2} \sin ^{2} \theta}, \\
a_{12} & =-\frac{\nu_{1}^{2} \nu_{2}^{2}\left(1-\nu_{1}^{2}\right)^{2}\left(1-\nu_{2}^{2}\right)^{2}\left(2-\nu_{1}^{2}-\nu_{2}^{2}\right)}{4\left(1+\nu_{1}^{4} \nu_{2}^{2}+\nu_{1}^{2} \nu_{2}^{4}-3 \nu_{1}^{2} \nu_{2}^{2}\right)^{2}} \frac{\sin ^{2} \theta \cos ^{2} \theta}{1-\nu_{1}^{2} \cos ^{2} \theta-\nu_{2}^{2} \sin ^{2} \theta}, \\
b^{2} & =\frac{\left(2-\nu_{1}^{2}-\nu_{2}^{2}\right)\left(1-\nu_{1}^{2} \cos ^{2} \theta-\nu_{2}^{2} \sin ^{2} \theta\right)}{4\left(1+\nu_{1}^{4} \nu_{2}^{2}+\nu_{1}^{2} \nu_{2}^{4}-3 \nu_{1}^{2} \nu_{2}^{2}\right)}
\end{aligned}
$$

with

$$
\mu_{1}^{2}=\frac{\nu_{1}^{2}\left(1-\nu_{1}^{2} \nu_{2}^{2}\right)}{2-\nu_{1}^{2}-\nu_{2}^{2}}, \quad \mu_{2}^{2}=\frac{\nu_{2}^{2}\left(1-\nu_{1}^{2} \nu_{2}^{2}\right)}{2-\nu_{1}^{2}-\nu_{2}^{2}} .
$$

The metric (2.36) can be regarded as one on the associated $S^{3}$-bundle of the principal $T^{2}$-bundle over $S^{2}$ with the Euler classes $\left(k_{1}, k_{2}\right) \in H^{2}\left(S^{2}, \mathbb{Z}\right)^{\oplus 2}=\mathbb{Z} \oplus \mathbb{Z}$. The invariant $[\gamma] \in \mathbb{Z}_{2}$ of the $S^{3}$-bundle is given by

$$
[\gamma]=k_{1}+k_{2} \bmod 2
$$

The connection $\omega=\omega^{1} \oplus \omega^{2}$ is given by

$$
\omega^{i}=d \psi_{i}+\cos \chi d \eta, \quad 0 \leq \psi_{i} \leq 4 \pi /\left|k_{i}\right|
$$

where we have rescaled the torus angles as $\psi_{i} \mapsto k_{i} \psi_{i}$.

Summarizing the consideration above, we state

Theorem 1. Let $\nu_{1}$ and $\nu_{2}$ be real numbers in the region $\nu_{1}^{2}, \nu_{2}^{2}>1$ and $\nu_{1}^{2} \neq \nu_{2}^{2}$ together with the integral conditions;

$$
\begin{aligned}
& k_{1}=\frac{\nu_{1}\left(1-\nu_{2}^{2}\right)\left(2-\nu_{2}^{2}-\nu_{1}^{2} \nu_{2}^{2}\right)}{1+\nu_{1}^{4} \nu_{2}^{2}+\nu_{1}^{2} \nu_{2}^{4}-3 \nu_{1}^{2} \nu_{2}^{2}}, \\
& k_{2}=\frac{\nu_{2}\left(1-\nu_{1}^{2}\right)\left(2-\nu_{1}^{2}-\nu_{1}^{2} \nu_{2}^{2}\right)}{1+\nu_{1}^{4} \nu_{2}^{2}+\nu_{1}^{2} \nu_{2}^{4}-3 \nu_{1}^{2} \nu_{2}^{2}},
\end{aligned}
$$

where $\left(k_{1}, k_{2}\right) \in \mathbb{Z} \oplus \mathbb{Z}$. Then, $\left\{g_{\nu_{1} \nu_{2}}\right\}$ gives an infinite series of inhomogeneous Einstein metrics with positive scalar curvature $20\left(1-\nu_{1}^{2} \nu_{2}^{2}\right) /\left(2-\nu_{1}^{2}-\nu_{2}^{2}\right)$ on $S^{3}$-bundles over $S^{2}$. If the integer $k_{1}+k_{2}$ is even (odd), then the bundle is trivial (non-trivial).

Remark 2. The region $\nu_{1}>1, \nu_{2}>1$ is mapped diffeomorphically onto the region $k_{1}>0, k_{2}>0, k_{1}+k_{2}>2$ by (2.45) and (2.46). Hence, there exists the unique pair $\left(\nu_{1}, \nu_{2}\right)$ for each $\left(k_{1}, k_{2}\right) \in S, S=\left\{\left(k_{1}, k_{2}\right) \in \mathbb{Z} \oplus \mathbb{Z} \mid k_{1} \neq \pm k_{2}, k_{1} \neq 0, k_{2} \neq 0\right\}$. For example, they are numerically evaluated as

(i) $\left(k_{1}, k_{2}\right)=(1,2), \quad\left(\nu_{1}, \nu_{2}\right)=(3.31133,2.14921)$,

(ii) $\left(k_{1}, k_{2}\right)=(1,3), \quad\left(\nu_{1}, \nu_{2}\right)=(7.68872,3.06769)$, 
(iii) $\left(k_{1}, k_{2}\right)=(2,3), \quad\left(\nu_{1}, \nu_{2}\right)=(5.85109,4.13646)$.

Remark 3. In the limit $\left(\nu_{1}, \nu_{2}\right)=\left(\nu_{1}, \infty\right)$, the metric tends to

$$
g_{\nu_{1} \infty}=d \theta^{2}+\frac{\sin ^{2} \theta}{4}\left(d \psi_{1}+\cos \chi d \eta\right)^{2}+\frac{\cos ^{2} \theta}{4} d \psi_{2}^{2}+\frac{\sin ^{2} \theta}{4} g_{S^{2}},
$$

after the rescaling $\nu_{1}^{2} g \rightarrow g$. When we make a modification of the range of $\psi_{1}$,

$$
0 \leq \psi_{1} \leq 4 \pi /\left|k_{1}\right| \longmapsto 0 \leq \psi_{1} \leq 4 \pi, \quad\left(k_{1}=\frac{1+\nu_{1}^{2}}{\nu_{1}}\right)
$$

then (2.47) represents the standard metric on $S^{5}$.

\section{Case B}

The parameters $\nu_{1}$ and $\nu_{2}$ are restricted to $0 \leq \nu_{1}^{2}, \nu_{2}^{2} \leq 1$ and $\nu_{1}^{2} \neq \nu_{2}^{2}$. By (2.22) we find that $0 \leq\left|k_{i}\right| \leq 2(i=1,2)$, hence there are two possibilities for $\left(\left|k_{1}\right|,\left|k_{2}\right|\right)$ under the integral condition ${ }^{\diamond}$ : (i) $\left(\left|k_{1}\right|,\left|k_{2}\right|\right)=(1,0)$, and (ii) $\left(\left|k_{1}\right|,\left|k_{2}\right|\right)=(2,0)$.

The case (ii) corresponds to $\left(\nu_{1}, \nu_{2}\right)=\left( \pm 1, \nu_{2}\right)$. The corresponding Einstein metric (2.19) is independent of $\nu_{2}$ and coincides with the standard $S^{5}$-metric after a modification of the angle $\psi_{1}=2 \tilde{\psi}_{1}\left(0 \leq \tilde{\psi}_{1} \leq 4 \pi\right)$ :

$$
g=d \theta^{2}+\frac{1}{4} \sin ^{2} \theta\left(d \tilde{\psi}_{1}+\cos \chi d \eta\right)^{2}+\frac{1}{4} \cos ^{2} \theta d \psi_{2}^{2}+\frac{1}{4} \sin ^{2} \theta g_{S^{2}} .
$$

One can show that the analysis remains true even if the range is extended to $\nu_{2}>1$.

In the case (i), we have $\left(\nu_{1}, \nu_{2}\right)=\left( \pm \frac{1}{2}, 0\right)$. Then, the Einstein metric (2.19) is of the form

$$
\begin{aligned}
g= & \frac{1-\frac{1}{4} \cos ^{2} \theta}{1-\frac{1}{7} \cos ^{2} \theta} d \theta^{2}+\left(\frac{7}{16}\right)^{2} \frac{1-\frac{1}{7} \cos ^{2} \theta}{1-\frac{1}{4} \cos ^{2} \theta} \sin ^{2} \theta\left(d \psi_{1}+\cos \chi d \eta\right)^{2} \\
& +\frac{1}{4} \cos ^{2} \theta d \psi_{2}^{2}+\frac{7}{16}\left(1-\frac{1}{4} \cos ^{2} \theta\right) g_{S^{2}},
\end{aligned}
$$

which gives a metric of cohomogeneity one with principal orbits $S^{3} \times S^{1}$. The principal orbits collapse to $S^{2} \times S^{1}$ at $\theta=0$ and $S^{3} \times\{$ point $\}$ at $\theta=\pi / 2$. Globally the metric can be regarded as one on the non-trivial $S^{3}$-bundle over $S^{2}$. In section 2, we will construct Einstein metrics on $S^{N}$-bundles over $S^{2}$ (see Theorem 3), generalizing the metric (2.50) to higher dimensions.

\section{Case C}

When we put $\nu_{1}=\nu_{2} \equiv \nu$, the metric (2.19) is ${ }^{\sharp}$

$$
g_{\nu}=d \theta^{2}+\sum_{i, j=1,2}^{2} a_{i j}(\theta) \omega^{i} \otimes \omega^{j}+\frac{2+\nu^{2}}{4\left(2 \nu^{2}+1\right)} g_{S^{2}}
$$

${ }^{\diamond}$ Remember that the metric is symmetric under $\left(\psi_{1}, \nu_{1}\right) \leftrightarrow\left(\psi_{2}, \nu_{2}\right)$.

${ }^{\sharp}$ We have rescaled the metric as $\frac{2+\nu^{2}}{2} g \rightarrow g$. 
where

$$
\begin{aligned}
& a_{11}=\frac{\sin ^{2} \theta}{4\left(2+\nu^{2}\right)}\left(2+\nu^{2} \cos ^{2} \theta\right), \\
& a_{22}=\frac{\cos ^{2} \theta}{4\left(2+\nu^{2}\right)}\left(2+\nu^{2} \sin ^{2} \theta\right), \\
& a_{12}=-\frac{\nu^{2}}{4\left(2+\nu^{2}\right)} \sin ^{2} \theta \cos ^{2} \theta .
\end{aligned}
$$

The 1-form $\omega^{1} \oplus \omega^{2}$ is a connection on the $T^{2}$-bundle over $S^{2}$, locally written as

$$
\omega^{i}=d \psi_{i}+k \cos \chi d \eta \quad\left(0 \leq \psi_{i} \leq 4 \pi\right) .
$$

Here the coefficient $k$ is evaluated as

$$
k=\frac{\nu\left(\nu^{2}+2\right)}{2 \nu^{2}+1}
$$

by using (2.22), and it is required to be $k \in \mathbb{Z}$. In this case the $T^{2}$-bundles collapse to the same lens space $L_{k}$ at each boundary. Notice that by introducing the Maurer-Cartan forms of $\mathrm{SU}(2)$

$$
\begin{aligned}
\sigma_{1} & =2 \cos \left(\frac{\psi_{1}+\psi_{2}}{2}\right) d \theta-\sin \left(\frac{\psi_{1}+\psi_{2}}{2}\right) \sin \theta \cos \theta\left(d \psi_{1}-d \psi_{2}\right), \\
\sigma_{2} & =-2 \sin \left(\frac{\psi_{1}+\psi_{2}}{2}\right) d \theta-\cos \left(\frac{\psi_{1}+\psi_{2}}{2}\right) \sin \theta \cos \theta\left(d \psi_{1}-d \psi_{2}\right), \\
\sigma_{3} & =\sin ^{2} \theta d \psi_{1}+\cos ^{2} \theta d \psi_{2},
\end{aligned}
$$

the fiber metric of (2.51) can be rewritten as

$$
g_{F}=d \theta^{2}+\sum_{i, j=1}^{2} a_{i j}(\theta) d \psi_{i} \otimes d \psi_{j}=\frac{1}{4}\left(\sigma_{1}^{2}+\sigma_{2}^{2}\right)+\frac{1}{2\left(2+\nu^{2}\right)} \sigma_{3}^{2},
$$

which reveals the $\mathrm{SU}(2)$ isometry of the metric.

Theorem 2. Let $\nu_{k}$ be real numbers satisfying $k=\nu_{k}\left(\nu_{k}^{2}+2\right) /\left(2 \nu_{k}^{2}+1\right) \in \mathbb{Z}$. Then, $\left\{g_{\nu_{k}}\right\}$ gives an infinite series of homogeneous Einstein metrics with positive scalar curvature $20\left(1+\nu_{k}^{2}\right) /\left(2+\nu_{k}^{2}\right)$ on $S^{2} \times S^{3}$.

proof. By the coordinate transformation,

$$
\alpha=2 \theta, \quad \beta=\frac{1}{2}\left(\psi_{2}-\psi_{1}\right) \text { and } t=\frac{1}{2}\left(\psi_{1}+\psi_{2}\right),
$$

the metric takes the form

$$
\begin{aligned}
g_{\nu_{k}}= & \frac{1}{4}\left(d \alpha^{2}+\sin ^{2} \alpha d \beta^{2}\right)+\frac{2+\nu^{2}}{4\left(2 \nu^{2}+1\right)}\left(d \chi^{2}+\sin ^{2} \chi d \eta^{2}\right) \\
& +\frac{1}{2\left(2+\nu^{2}\right)}(d t+\cos \alpha d \beta+k \cos \chi d \eta)^{2}
\end{aligned}
$$


which represents a Kaluza-Klein metric on the total space $M_{k, 1}^{1,1}$ of the circle bundle over $\mathbb{C} P^{1} \times \mathbb{C} P^{1}$ with Euler class $e=k \alpha_{1}+\alpha_{2}$, where $\alpha_{1}$ and $\alpha_{2}$ are generators in $H^{2}\left(\mathbb{C} P^{1} \times\right.$ $\left.\mathbb{C} P^{1} ; \mathbb{Z}\right)=\mathbb{Z} \oplus \mathbb{Z}$. The space $M_{k, 1}^{1,1}$ is diffeomorphic to $S^{2} \times S^{3}[7$.

Remark 4. There exists the unique real number $\nu_{k}$ for each $k \in \mathbb{Z}$. The value of $\nu_{k}$ is explicitly given by

$$
\begin{aligned}
\nu_{k} & =\frac{1}{6}\left[a^{1 / 3}-8\left(3-2 k^{2}\right) a^{-1 / 3}+4 k\right], \\
a & =-36 k+64 k^{3}+12 \sqrt{96-183 k^{2}+96 k^{4}}
\end{aligned}
$$

for $k \geq 0$, and $\nu_{-k}=-\nu_{k}$.

Remark 5. In the case $\nu_{0}=0$, the metric coincides with the product metric on $S^{2} \times S^{3}$ :

$$
g_{0}=d \theta^{2}+\frac{1}{4} \sin ^{2} \theta d \psi_{1}^{2}+\frac{1}{4} \cos ^{2} \theta d \psi_{2}^{2}+\frac{1}{2} g_{S^{2}} .
$$

On the other hand, in the limit $\nu_{k} \rightarrow \pm \infty(k \rightarrow \pm \infty)$, the fiber $S^{1}$ of $S^{2} \times S^{3} \rightarrow S^{2} \times S^{2}$ collapses, and the metric tends to the product Riemannian metric on $S^{2} \times S^{2}$ which is not Einstein:

$$
g_{\infty}=d \theta^{2}+\left(\frac{\sin \theta \cos \theta}{2}\right)^{2} d \psi_{-}^{2}+\frac{1}{8} g_{S^{2}}
$$

with $\psi_{-}=\psi_{1}-\psi_{2}$.

\section{$3 \quad d$-dimensional Einstein Metrics}

The AdS Kerr black hole in $d$-dimensions $(d \geq 4)$ was constructed in [4. It can be straightforwardly transformed to the Euclidean form,

$$
\begin{aligned}
g_{d}= & \frac{\Delta_{r}}{\rho^{2}}\left(d \tau-\frac{\alpha}{\Xi} \sin ^{2} \theta d \phi\right)^{2}+\frac{\Delta_{\theta} \sin ^{2} \theta}{\rho^{2}}\left(\alpha d \tau+\frac{r^{2}-\alpha^{2}}{\Xi} d \phi\right)^{2} \\
& +\frac{\rho^{2}}{\Delta_{r}} d r^{2}+\frac{\rho^{2}}{\Delta_{\theta}} d \theta^{2}+r^{2} \cos ^{2} \theta g_{S^{d-4}},
\end{aligned}
$$

where $g_{S^{d-4}}$ is the standard metric on $S^{d-4}$ with the positive scalar curvature $(d-4)(d-5)$, which is Einstein ${ }^{b}$, and

$$
\begin{aligned}
\rho^{2} & =r^{2}-\alpha^{2} \cos ^{2} \theta \\
\Delta_{r} & =\left(r^{2}-\alpha^{2}\right)\left(1-l^{2} r^{2}\right)-2 M r^{5-d} \\
\Delta_{\theta} & =1-\alpha^{2} l^{2} \cos ^{2} \theta
\end{aligned}
$$

${ }^{b}$ The sphere metric $g_{S^{d-4}}$ can be replaced by $g_{M_{d-4}}$, where $g_{M_{d-4}}$ is an arbitrary Einstein metric on a $(d-4)$-dimensional manifold with the positive scalar curvature $(d-4)(d-5)$. 
with the parameter $\Xi=1-\alpha^{2} l^{2}$. We find that there exists a double root $r_{0}$ of $\Delta_{r}=0$, when the following condition for the parameters is satisfied:

$$
\begin{aligned}
r_{0} & =\left[\frac{d-3-(d-5) \nu^{2}}{d-1-(d-3) \nu^{2}}\right]^{1 / 2} l^{-1}, \\
M_{0} & =\frac{(1-\nu)^{2}}{d-1-(d-3) \nu^{2}}\left[\frac{d-3-(d-5) \nu^{2}}{d-1-(d-3) \nu^{2}}\right]^{\frac{d-3}{2}} l^{-(d-3)}, \\
\Xi_{0} & =1-\frac{\nu^{2}\left(d-3-(d-5) \nu^{2}\right)}{d-1-(d-3) \nu^{2}},
\end{aligned}
$$

where we have introduced a dimensionless parameter $\nu=\alpha / r_{0}$. Then, $\Delta_{r}$ takes the form

$$
\begin{aligned}
\Delta_{r} & =-\left(r-r_{0}\right)^{2} \tilde{\Delta}(r), \\
\tilde{\Delta}(r) & =\frac{1}{r^{d-5}}\left(c_{0}+c_{1} r+\cdots+c_{d-3} r^{d-3}\right),
\end{aligned}
$$

where

$$
\begin{aligned}
c_{i} & =\frac{2(1+i)\left(1-\nu^{2}\right)^{2}}{d-1-(d-3) \nu^{2}} r_{0}^{d-i-5} \quad(0 \leq i \leq d-6), \\
c_{d-5} & =\frac{2 d-8-3(d-5) \nu^{2}+(d-5) \nu^{4}}{d-1-(d-3) \nu^{2}} \\
c_{d-4} & =2 r_{0} l^{2} \\
c_{d-3} & =l^{2}
\end{aligned}
$$

The remaining procedure is completely parallel to the one in the section 2 ; consider a nearly extreme black hole, and take the limit $\varepsilon \rightarrow 0$. Actually we define new coordinates $\left(\eta, \chi, \phi_{1}\right)$ instead of $(\tau, r, \phi)$ as

$$
\begin{aligned}
r & =r_{0}-\varepsilon \cos \chi \\
\eta & =\frac{\varepsilon \tilde{\Delta}\left(r_{0}\right)}{r_{0}^{2}\left(1-\nu^{2}\right)} \tau+O\left(\varepsilon^{2}\right), \\
\phi_{1} & =\phi+\frac{\alpha \Xi}{r_{1}^{2}-\alpha^{2}} \quad\left(r_{1} \equiv r_{0}-\varepsilon\right),
\end{aligned}
$$

where $\tilde{\Delta}\left(r_{0}\right)$ can be calculated by (3.9);

$$
\tilde{\Delta}\left(r_{0}\right)=\frac{(d-5)(d-3) \nu^{4}-2(d-1)(d-5) \nu^{2}+(d-1)(d-3)}{d-1-(d-3) \nu^{2}} .
$$

In the limit $\varepsilon \rightarrow 0$, we find a one-parameter family of $d$-dimensional Einstein metrics

$$
g_{\nu}=h^{2}(\theta) d \theta^{2}+\sum_{i=1}^{3} a_{i}(\theta) \sigma^{i} \otimes \sigma^{i}+b^{2}(\theta) g_{S^{d-4}} .
$$


with the positive scalar curvature $d(d-1)\left(d-3-(d-5) \nu^{2}\right) /\left(d-1-(d-3) \nu^{2}\right)$. Here $\sigma^{i}(i=1,2,3)$ are 1 -forms defined by

$$
\begin{aligned}
\sigma^{1} & =\cos \psi d \chi+\sin \psi \sin \chi d \eta \\
\sigma^{2} & =-\sin \psi d \chi+\cos \psi \sin \chi d \eta \\
\sigma^{3} & =d \psi+k \cos \chi d \eta
\end{aligned}
$$

with $\phi_{1}=\frac{1}{2}(\psi+k \eta)$ and

$$
k=\frac{4 \nu\left(d-1-(d-5) \nu^{2}\right)}{(d-5)(d-3) \nu^{4}-2(d-1)(d-5) \nu^{2}+(d-1)(d-3)} .
$$

The metric components are found to be

$$
\begin{aligned}
h^{2} & =\frac{1-\nu^{2} \cos ^{2} \theta}{1-\mu^{2} \cos ^{2} \theta}, \\
a_{1} & =a_{2}=\frac{1-\nu^{2} \cos ^{2} \theta}{\tilde{\Delta}}, \\
a_{3} & =\frac{1}{4}\left(\frac{1-\nu^{2}}{1-\mu^{2}}\right)^{2} \frac{1-\mu^{2} \cos ^{2} \theta}{1-\nu^{2} \cos ^{2} \theta} \sin ^{2} \theta, \\
b^{2} & =\cos ^{2} \theta,
\end{aligned}
$$

where

$$
\mu^{2}=\frac{(d-3) \nu^{2}-(d-5) \nu^{4}}{d-1-(d-3) \nu^{2}}
$$

and $\tilde{\Delta}$ is given by (3.17).

To avoid singularities, we will assume $0 \leq \nu^{2} \leq 1$. In this range, $\mu^{2}$ and $k$ are monotonously increasing functions with respect to $\nu$. Then, we have $0 \leq \mu^{2} \leq 1,-2 \leq$ $k \leq 2$ and $\tilde{\Delta}>0(d \geq 4)$. By the analysis similar to section 2 , the ranges of angles must be restricted as $0 \leq \theta \leq \frac{\pi}{2}, 0 \leq \psi \leq 4 \pi, 0 \leq \chi \leq \pi$ and $0 \leq \eta \leq 2 \pi$. If we impose that $k \in \mathbb{Z}$, the 1 -form $\sigma^{3}$ can be regarded as a connection on the lens space $L_{k}$. Taking account of the inequality $|k| \leq 2$, we have

$$
\text { (i) }|k|=0, \quad \text { (ii) }|k|=1, \quad \text { (iii) }|k|=2 .
$$

The case (i) corresponds to $\nu=0$, and then the metric (3.18) gives the product metric on $S^{2} \times S^{d-2}$;

$$
g_{0}=\frac{1}{d-3}\left(\left(\sigma^{1}\right)^{2}+\left(\sigma^{2}\right)^{2}\right)+d \theta^{2}+\frac{1}{4} \sin ^{2} \theta d \psi^{2}+\cos ^{2} \theta g_{S^{d-4}} .
$$

The case (iii) corresponds to $\nu= \pm 1$, and then the metric (3.18) is the standard $S^{d}$-metric after a modification of the angle $\psi \rightarrow 2 \psi$;

$$
g_{ \pm 1}=d \theta^{2}+\frac{1}{4} \sin ^{2} \theta\left(\left(\sigma^{1}\right)^{2}+\left(\sigma^{2}\right)^{2}+\left(\sigma^{3}\right)^{2}\right)+\cos ^{2} \theta g_{S^{d-4}}
$$


In the case (ii), the 1 -forms $\sigma^{i}(i=1,2,3)$ are identified with the Maurer-Cartan forms of $\mathrm{SU}(2)$. Thus the metric is of cohomogeneity one with principal orbits $S^{3} \times S^{d-4}$. The orbits collapse to $S^{2} \times S^{d-4}$ at $\theta=0$ and to $S^{3} \times\{$ point $\}$ at $\theta=\pi / 2$. Hence, the total space is the unit sphere bundle of the vector bundle $H \oplus \underline{\mathbb{R}}^{d-3}$ over $S^{2}$, where $H$ is the Hopf bundle and $\underline{\mathbb{R}}^{d-3}$ is the trivial bundle of rank $d-3$. Since the second Stiefel-Whitney class $w_{2}\left(H \oplus \underline{\mathbb{R}}^{d-3}\right)=w_{2}(H) \neq 0$ in $H^{2}\left(S^{2} ; \mathbb{Z}\right)=\mathbb{Z}_{2}$, the space is $S^{2} \widetilde{\times} S^{d-2}$, the non-trivial $S^{d-2}$-bundle over $S^{2}$.

Theorem 3 Let $\nu$ be a real number satisfying $\nu^{2}<1$ and

$$
\frac{4 \nu\left(d-1-(d-5) \nu^{2}\right)}{(d-5)(d-3) \nu^{4}-2(d-1)(d-5) \nu^{2}+(d-1)(d-3)}= \pm 1 .
$$

Then $g_{\nu}$ gives an Einstein metric with positive scalar curvature on $S^{d-2} \widetilde{\times} S^{2}$, which is the non-trivial $S^{d-2}$-bundle over $S^{2}$.

Remark 6. For $d=4$, this result reproduces the Page metric on $\mathbb{C} P^{2} \sharp \overline{\mathbb{C} P^{2}}$. In this case the principal orbits are $S^{3}$, the range of $\theta$ is extended to $0 \leq \theta \leq \pi$ and the metric has $\mathbb{Z}_{2}$-symmetry about $\theta=\pi / 2$. For $d=5$, this represents the metric (2.50).

\section{Acknowledgements}

We thank R. Goto, H. Ishihara and S. Tanimura for useful discussions. Y.Y. would like to express his gratitude to G.W. Gibbons and S.A. Hartnoll for useful discussions during his stay at DAMTP, Cambridge University. This paper is supported by the 21 COE program "Constitution of wide-angle mathematical basis focused on knots". Research of Y.H. is supported in part by the Grant-in Aid for scientific Research (No. 15540090) from Japan Ministry of Education. Research of Y.Y. is supported in part by the Grant-in Aid for scientific Research (No. 14540073 and No. 14540275) from Japan Ministry of Education.

\section{References}

[1] J. M. Maldacena, "The large N limit of superconformal field theories and supergravity," Adv. Theor. Math. Phys. 2 (1998) 231 [Int. J. Theor. Phys. 38 (1999) 1113 arXiv:hep-th/9711200; S. S. Gubser, I. R. Klebanov and A. M. Polyakov, "Gauge theory correlators from non-critical string theory," Phys. Lett. B 428 (1998) 105 arXiv:hep-th/9802109; E. Witten, "Anti-de Sitter space and holography," Adv. Theor. Math. Phys. 2 (1998) 253 arXiv:hep-th/9802150. 
[2] S. W. Hawking and D. N. Page, "Thermodynamics Of Black Holes In Anti-De Sitter Space," Commun. Math. Phys. 87 (1983) 577.

[3] E. Witten, "Anti-de Sitter space, thermal phase transition, and confinement in gauge theories," Adv. Theor. Math. Phys. 2 (1998) 505 arXiv:hep-th/9803131.

[4] S. W. Hawking, C. J. Hunter and M. M. Taylor-Robinson, "Rotation and the AdS/CFT correspondence," Phys. Rev. D 59 (1999) 064005 arXiv:hep-th/9811056.

[5] D. Page, "A Compact Rotating Gravitational Instanton," Phys. Lett. B 79 (1978) 235.

[6] C. Böhm, "Inhomogeneous Einstein metrics on low-dimensional spheres and other low-dimensional spaces," Invent. math. 134, (1998) 145-176.

[7] M. Y. Wang and W. Ziller, "Einstein metrics on principal torus bundles", J. Diff. Geom. 31 (1990) 215-248. 